\title{
Conhecimento, atitude e prática de adolescentes escolares em relação ao uso do preservativo masculino
}

\author{
Knowledge, attitude and practice \\ of school teenagers in relation to \\ the use of male condoms
}

\section{Joyce Mazza Nunes Aragão' (1) Hiara Rose Moreno Amaral ${ }^{2}$ (1) Francisco Willian Melo de Sousa ${ }^{3}$ (1) Eliany Nazaré Oliveira ${ }^{4}$ (1) Maristela Inês Osawa Vasconcelos ${ }^{5}$ (1)}

1'Autora principal para correspondência. Universidade Estadual Vale do Acaraú (Sobral). Ceará, Brasil. joycemazza@hotmail.com
${ }^{2-5}$ Universidade Estadual Vale do Acaraú (Sobral). Ceará, Brasil. hiara_amaral@hotmail.com, williancarire123@gmail.com, elianyy@hotmail.com, miosawa@gmail.com

\begin{abstract}
RESUMO | OBJETIVO: Avaliar conhecimentos, atitudes e práticas de adolescentes escolares em relação ao preservativo masculino como método contraceptivo de primeira escolha para os adolescentes. MÉTODO: Estudo com abordagem quantitativa, realizado com 143 adolescentes de uma escola estadual de ensino médio, no período de outubro a novembro de 2019. Para a coleta de dados, aplicou-se o inquérito Conhecimento, Atitude e Prática (CAP) sobre o uso do preservativo masculino. RESULTADOS: Evidenciou-se que 133 (93\%) dos adolescentes apresentaram conhecimento inadequado, 105 (73,4\%) atitude inadequada e $130(90,9 \%)$ prática inadequada a respeito do uso do preservativo masculino. Esses desfechos se mostram preocupantes, uma vez que a atividade sexual na adolescência está cada vez mais precoce, gerando, também, maior exposição a riscos, como IST/HIV/Aids e gravidez indesejada, principalmente quando esta prática é realizada sem uso ou com uso inadequado do preservativo. CONCLUSÃO: As fragilidades nas informações obtidas pelos adolescentes refletem o conhecimento inadequado pela quase totalidade deles, seguidas pela atitude e prática inadequadas quanto ao uso do preservativo masculino. Dessa forma, visualiza-se a necessidade de potencializar estratégias educativas, com intuito de ampliar o conhecimento de adolescentes acerca do uso do preservativo masculino e, consequentemente, desenvolver prática adequada desse método contraceptivo.
\end{abstract}

DESCRITORES: Saúde do Adolescente. Preservativos. Conhecimentos. Atitudes e Prática em Saúde. Comportamento sexual.

\begin{abstract}
OBJECTIVE: Evaluate the knowledge, attitudes, and practices of school adolescents concerning the male condom as a contraceptive method of the first choice for adolescents. METHODOLOGY: A study with a quantitative approach carried out with 143 adolescents from a state high school during October and November 2019. As a data collection instrument, the Knowledge, Attitude, and Practice (CAP) survey was applied to using the male condom. RESULTS: It was evidenced that $133(93 \%)$ of the adolescents presented inadequate knowledge, 105 (73.4\%) inadequate attitude and $130(90.9 \%)$ inadequate practice regarding the use of the male condom. These outcomes are worrisome since sexual activity in adolescence is increasingly precocious, generating greater exposure to risks such as STI / HIV / AIDS and unwanted pregnancy, mainly when this practice is performed without use or with the inappropriate use of condoms. CONCLUSION: The weaknesses in the information obtained by the adolescents, reflected in their inadequate knowledge, followed by the inadequate attitude and practice regarding the use of the male condom. Thus, there is a need to enhance educational strategies to expand adolescents' knowledge about the use of male condoms, and consequently, develop a good practice of this contraceptive method.
\end{abstract}

DESCRIPTORS: Adolescent Health. Condoms. Health Knowledge. Attitudes and Practice in health. Sexual behavior. 


\section{Introdução}

A adolescência é uma fase que representa a transição entre a infância e a vida adulta, na qual é marcada por modificações biopiscosociais. Neste ínterim, os adolescentes estão em constantes mudanças e descobertas, sejam biológicas, nas relações familiares, sociais e sexuais ${ }^{1}$. Segundo a Organização Mundial de Saúde (OMS), este período compreende entre 10 e 19 anos, já de acordo com o Estatuto da Criança e do Adolescente (ECA), no Brasil, essa etapa é definida entre 12 e 18 anos $^{2}$.

Dentre as mudanças dessa fase, têm-se as relacionadas à sexualidade e as transformações psicológicas e corporais decorrentes da puberdade ${ }^{3}$. Além disso, nesse período, os adolescentes estão sujeitos à exposição diante dos comportamentos nocivos à saúde, como uso de álcool, tabagismo, maus hábitos alimentares, início precoce e desprotegido da atividade sexual, desencadeando, assim, riscos e adoecimento à saúde física e mental, além de conflitos nas relações interfamiliar e social ${ }^{1}$.

Para os adolescentes, a escola é um espaço de compromisso social e estratégico para o diálogo e a orientação quanto aos aspectos relacionados ao conhecimento e à mudança de comportamento, pois o jovem se encontra boa parte do tempo neste local ${ }^{4}$. Nesta perspectiva, é no ambiente escolar que, muitas vezes, os aspectos de caráter biológico, emocional e psicológico se afloram no adolescente. Assim, a escola é um local ideal para construção das relações humanas, esclarecimento de curiosidades e dúvidas e, dentre estas, pode-se destacar as preocupações com o corpo 5 .

A gravidez na adolescência é outro problema de saúde pública, haja vista os riscos que o processo gravídico nessa fase pode desencadear ao binômio mãe/ filho, bem como por estar associado com o início precoce da atividade sexual e as chances de mais gestações futuras ${ }^{6}$. Nesta perspectiva, dentre as consequências negativas desse evento para a adolescente, destacam-se: mudança de papéis e comportamento; desenvolvimento e crescimento precoce do corpo; interrupção do processo educacional; e complicações durante e pós-parto. Já para o bebê, enfatizam-se os riscos advindos do parto prematuro ${ }^{\text {. }}$.
Destarte, apesar das consequências que a gravidez acarreta na adolescência, evidencia-se que esse evento é crescente em âmbito nacional. Estudo aponta que a taxa de gestação desse grupo no Brasil é alta para a América Latina, em 2015, por exemplo, 18\% eram filhos de mães adolescentes ${ }^{8}$. Dentre os fatores que podem contribuir para isso, destacam-se conhecimento deficiente acerca do uso de métodos contraceptivos, os aspectos socioculturais e a associação com consumo de álcool e uso do cigarro ${ }^{3}$.

Nesse sentido, a orientação sobre sexualidade é fundamental para o público adolescente, pois, em muitos casos, estes não conseguem nem mesmo identificar as mudanças ocorridas durante a puberdade. Vinculado a isso, pode estar associada à incapacidade de reconhecimento de sinais e sintomas de transmissão de qualquer Infecção Sexualmente Transmissível (IST). O não reconhecimento de sintomas, associado com a aparência saudável, pode influenciar o hábito sexual sem prevenção adequada, aumentando as chances de disseminação de IST? ${ }^{2}$.

Estudo realizado com estudantes adolescentes da rede pública de Cuiabá/MT, a respeito do conhecimento dos métodos contraceptivos e respectivo uso, evidenciou déficit de conhecimento acerca da utilização da camisinha masculina e feminina, do anticoncepcional oral e da pílula do dia seguinte ${ }^{10}$. Outro estudo acerca dessa temática, realizado com adolescentes colombianos revelou que dos entrevistados $65,6 \%$ conhecem os métodos anticoncepcionais, porém $54,7 \%$ disseram usar nas relações sexuais; o preservativo é o método mais utilizado pelos participantes. $O$ estudo ainda esclareceu que o desconhecimento e as crenças sobre os efeitos colaterais, aumento de peso e infertilidade, por exemplo, são fatores desencadeantes para o não uso dos métodos ${ }^{11}$.

Nesse contexto, estudos apontam lacunas no conhecimento de adolescentes acerca da saúde sexual e reprodutiva, bem como do acesso às orientações/informações fidedignas diante deste assunto ${ }^{10-12}$. Assim, tais aspectos justificam o desenvolvimento deste estudo que objetivou avaliar conhecimentos, atitudes e práticas de adolescentes escolares em relação ao preservativo masculino como método contraceptivo de primeira escolha para os adolescentes. 


\section{Método}

Trata-se de estudo de abordagem quantitativa, realizado com 143 adolescentes de uma escola estadual de ensino médio, da cidade de Sobral-CE, Brasil, durante os meses de outubro e novembro de 2019.

Utilizou-se da amostragem não probabilística por conveniência, a qual foi selecionada de acordo com os seguintes critérios de inclusão: estar na fase da adolescência, com idades de 10 aos 19 anos completos, conforme preconizado pela OMS, estar devidamente matriculado(a) na referida escola e cursando o primeiro ano do ensino médio, nos turnos manhã ou tarde. Os alunos(as) dessa série escolar foram selecionados porque concentram adolescentes na fase intermediária, ou seja, na faixa etária de 15 a 17 $\operatorname{anos}^{13}$ e porque geralmente é nesse período que se concentra a ocorrência da primeira relação sexual ${ }^{14}$. Excluíram-se os adolescentes que não aceitaram participar do estudo.

Realizou-se reunião inicial com todos os alunos das turmas envolvidas, quando foram esclarecidos quanto à natureza e aos objetivos do estudo, utilizandose de linguagem clara e adequada à compreensão, para convidá-los a participar do estudo. Após aceitarem, oficializou-se a participação em Termo de Consentimento Livre e Esclarecido (TCLE), que foi assinado pelo responsável legal, seguido do Termo de Assentimento, assinado pelos adolescentes. Somente os adolescentes maiores de 18 anos de idade assinaram o TCLE.

Antes de iniciar a coleta de dados, elaborou-se um cronograma, juntamente com coordenadores e professores da escola, no intuito de realizar a coleta de dados nos horários mais adequados para os escolares. Assim, o questionário foi aplicado presencialmente, por acadêmicos de enfermagem integrantes da equipe de pesquisadores do estudo, nas salas de aula das seis turmas, sendo três do turno da manhã e três do turno da tarde, com duração de 30 minutos.

Para identificação dos conhecimentos, das atitudes e práticas dos adolescentes em relação ao preservativo masculino, utilizou-se do Inquérito Conhecimento, Atitude e Prática (CAP) em relação ao preservativo masculino que, para tanto, foi adaptado de um modelo previamente testado em estudo similar, em relação ao preservativo masculino ${ }^{17}$. Esse instrumento é composto de oito questões: 1) você já ouviu falar sobre o preservativo? Você sabe para que serve o preservativo? 2) Cite pelo menos três cuidados necessários para o uso correto do preservativo. 3) $\mathrm{Na}$ sua opinião, quando se deve utilizar o preservativo na relação sexual vaginal, oral e anal? 4) Você utiliza o preservativo em suas relações sexuais? 5) Explique os motivos e as ocasiões da não utilização. 6) Como você utiliza o preservativo em suas relações sexuais? 7) Você já engravidou ou engravidou a parceira indesejadamente? 8) Você já foi diagnosticado(a) com alguma Infecção Sexualmente Transmissível (IST)?

O conhecimento é considerado adequado quando o adolescente tiver ouvido falar sobre o preservativo masculino, souber que é para prevenir as IST/HIV em geral e a gravidez indesejada e souber citar, pelo menos, três cuidados necessários para o uso correto do método. Para a atitude ser considerada adequada, o adolescente deve referir que é sempre necessário o uso do preservativo masculino em todas as práticas sexuais. Para a prática ser considerada adequada, o adolescente deve referir utilizar preservativo masculino sempre e do início ao fim das práticas sexuais realizadas; não ter engravidado ou engravidado a parceira indesejadamente; não ter sido diagnosticado (a) IST $\stackrel{15}{\text {. }}$.

Os inquéritos CAP possibilitam que se identifique o que uma população específica conhece, pensa e como faz, frente a algum problema, por meio de questionários sobre diferentes variáveis, viabilizando o comportamento, com intuito de utilizar intervenções eficazes futuramente, podendo se adaptar a diversificados contextos, visando potencializar planejamentos de promoção à saúde ${ }^{16}$.

Os adolescentes também preencheram outro questionário, elaborado pelos autores, que contemplava questões de múltipla escolha e dados referentes à caracterização sociodemográfica e de comportamento sexual (perfil sociodemográfico e de comportamento sexual), e ainda sobre o diálogo com pais e professores sobre saúde sexual e reprodutiva, com intuito de conhecer melhor os participantes, traçando um perfil destes. 
Procedeu-se à análise descritiva dos dados, utilizando-se do software Excel, organizando os resultados em tabelas, quando foram apontadas as frequências e percentuais, segundo as variáveis: conhecimento sobre saúde sexual e reprodutiva, atitude e prática sobre o uso do preservativo masculino, comportamento sexual dos adolescentes e classificação do CAP.

A pesquisa obedeceu à Resolução 466/201, do Conselho Nacional de Saúde, a qual envolve pesquisa com seres humanos, e foi aprovada pelo Comitê de Ética em Pesquisa (CEP) da Universidade Estadual Vale do Acaraú, conforme parecer n. 3.432 .975 e Certificado de Apresentação para Apreciação Ética n. 16237219.2.0000.5053.

\section{Resultados}

Quanto aos dados sociodemográficos dos participantes do estudo, evidenciou-se que todos os participantes estavam na faixa etária de 15 a 19 anos, predominando o sexo masculino 77 (53,8\%), autodeclarados da cor parda 89 (62,2\%), solteiros e sem parceiro fixo $78(54,5 \%)$ e que o chefe da família possuía ensino fundamental completo e/ou ensino médio incompleto $44(30,77 \%)$.

Sobre a renda familiar, $83(58,0 \%)$ não sabiam qual a renda familiar. Dentre aqueles que sabiam, 22 $(15,4 \%)$ referiram ser menos que um salário mínimo, e $19(13,3 \%)$ afirmaram renda igual ao salário mínimo.
Acerca da religião, $87(60,8 \%)$ professavam a religião católica. A maioria, 122 (85,3\%), residia com os pais e afirmou ter orientação sexual heterossexual, 117 $(81,8 \%)$.

Investigou-se também o conhecimento dos adolescentes sobre sexualidade, IST/HIV e preservativos, bem como os diálogos com pais, professores e amigos sobre saúde sexual e reprodutiva. Esses resultados estão apresentados na Tabela 1.

Referente aos assuntos mais abordados pelos pais com os adolescentes frente à questão da sexualidade $52(22,5 \%)$ referiram ser sobre a gravidez, enquanto o conteúdo mais abordado pelos professores $85(24,1 \%)$ relataram ser sobre sexualidade/sexo. A maioria $134(93,7 \%)$ relatou já ter ouvido falar sobre o uso do preservativo e 108 (75,5\%) sobre os dois tipos (masculino e feminino). Em relação à pessoa com quem mais dialogavam sobre o uso do preservativo, $49(21,4 \%)$ adolescentes mencionaram os amigos. Somente 13 (5,7\%) afirmaram conversar sobre essa temática com os profissionais de saúde (Tabela 1).

Ainda no que concerne ao conhecimento sobre saúde sexual e reprodutiva, especialmente relacionados ao uso do preservativo masculino, percebe-se que este diálogo está mais presente na escola, pois foi o local em que $80(28,2 \%)$ adolescentes referiram ter tido maiores informações. A maior frequência de estudantes 77 (53,9\%) citou que o preservativo servia para prevenir IST/HIV/AIDS e gravidez, embora 97 (67\%) não soubessem os cuidados que deveriam ter para o seu uso (Tabela 1). 
Tabela 1. Conhecimento sobre sexualidade, Infecções Sexualmente Transmissíveis (IST/HIV) e uso de preservativo entre adolescentes de uma escola estadual de ensino médio, Sobral-Ceará 2019 ( $n=143)$

\begin{tabular}{|c|c|c|}
\hline Variáveis & $\mathbf{N}$ & $\%$ \\
\hline \multicolumn{3}{|l|}{ Temática abordada pelos pais } \\
\hline Não conversam sobre o assunto & 66 & 28,6 \\
\hline Gravidez & 52 & 22,5 \\
\hline Uso do preservativo & 51 & 22,1 \\
\hline Sexualidade/sexo & 34 & 14,7 \\
\hline IST/HIV & 28 & 12,1 \\
\hline \multicolumn{3}{|l|}{ Temática abordada pelos professores } \\
\hline Não conversam sobre o assunto & 86 & 24,4 \\
\hline Sexualidade/sexo & 85 & 24,1 \\
\hline IST/HIV & 82 & 23,3 \\
\hline Uso do preservativo & 66 & 18,8 \\
\hline Gravidez & 33 & 9,4 \\
\hline \multicolumn{3}{|l|}{ Já ouviu falar sobre preservativo } \\
\hline Sim & 134 & 93,7 \\
\hline Não & 9 & 6,3 \\
\hline \multicolumn{3}{|c|}{ Tipo de preservativo sobre o qual já ouviu falar } \\
\hline Os dois (feminino e masculino) & 108 & 75,5 \\
\hline Só masculino & 26 & 18,2 \\
\hline Só feminino & 9 & 6,3 \\
\hline \multicolumn{3}{|c|}{ Com quem você mais conversa sobre o uso do preservativo? } \\
\hline Amigos & 49 & 21,4 \\
\hline Mãe & 40 & 17,4 \\
\hline Não converso isso com ninguém & 32 & 14,0 \\
\hline Namorado(a)/esposo(a) & 29 & 12,7 \\
\hline Professores & 29 & 12,7 \\
\hline Irrmãos & 18 & 7,8 \\
\hline Pai & 16 & 7,0 \\
\hline Profissionais de saúde & 13 & 5,7 \\
\hline Outros & 3 & 1,3 \\
\hline \multicolumn{3}{|c|}{ Onde obteve informações sobre o uso de preservativo? } \\
\hline Escola & 80 & 28,2 \\
\hline Amigos & 55 & 19,4 \\
\hline Família & 51 & 18,0 \\
\hline Posto de Saúde & 31 & 10,9 \\
\hline Televisão & 22 & 7,7 \\
\hline Redes sociais na internet & 18 & 6,3 \\
\hline Sites da Internet & 15 & 5,3 \\
\hline Outros & 8 & 2,8 \\
\hline Revistas & 4 & 1,4 \\
\hline \multicolumn{3}{|l|}{ Para que serve o preservativo? } \\
\hline Para prevenir IST/HIV/AIDS e gravidez & 77 & 53,8 \\
\hline Só para evitar gravidez & 29 & 20,3 \\
\hline Só para prevenir as IST/HIV & 18 & 12,6 \\
\hline Não sabe & 11 & 7,7 \\
\hline Para outras finalidades & 8 & 5,6 \\
\hline \multicolumn{3}{|c|}{ Quais os cuidados citados para o uso do preservativo? } \\
\hline Não sabe & 97 & 67,8 \\
\hline Não rasgar & 26 & 18,2 \\
\hline Não abrir com o dente & 15 & 10,5 \\
\hline Amarrar e jogar no local adequado & 5 & 3,5 \\
\hline
\end{tabular}

Fonte: Elaborado pelos autores, 2020. 
A Tabela 2 apresenta os dados referentes à atitude dos adolescentes sobre o uso do preservativo masculino.

O uso do preservativo durante o sexo oral foi apontado como necessário apenas por $49(34,3 \%)$ adolescentes. Constatou-se que $113(79,0 \%)$ adolescentes consideram que é necessário utilizar o preservativo durante o sexo vaginal, e pouco menos da metade, 68 (47,5\%), afirmou a necessidade de utilizar durante o sexo anal.

Tabela 2. Atitude de adolescentes de escola estadual de ensino médio em relação ao uso do preservativo masculino, Sobral-Ceará, 2019. (n=143)

\begin{tabular}{lcc}
\hline Variáveis & $\mathbf{N}$ & $\%$ \\
\hline Uso do preservativo no sexo vaginal & 113 & 79,0 \\
É necessário & 20 & 14,0 \\
Não tem opinião & 9 & 6,3 \\
É desnecessário & 1 & 0,7 \\
Não respondeu & 53 & 37,0 \\
Uso do preservativo no sexo oral & 49 & 34,3 \\
É desnecessário & 40 & 28,0 \\
É necessário & 1 & 0,7 \\
Não tem opinião & & \\
Não respondeu & 68 & 47,5 \\
Uso do preservativo no sexo anal & 39 \\
É necessário & 35 \\
É desnecessário & 1 & 27,3 \\
Não tem opinião & 24,5 \\
Não respondeu & & 0,7 \\
\hline
\end{tabular}

Fonte: Elaborado pelos autores, 2020.

A Tabela 3 apresenta o comportamento sexual de adolescentes do estudo, com ênfase na prática do uso do preservativo masculino.

A idade da primeira relação sexual variou entre 12 e 17 anos, sendo que a maioria, 29 (20,3\%), iniciou a atividade sexual aos 15 anos de idade. A primeira relação sexual dos adolescentes foi mencionada por $46(30,1 \%)$ que aconteceu com o namorado(a), ter utilizado o preservativo masculino na primeira relação sexual por 38 (26,6\%), que ainda não tinham filhos por 128 (89,5\%), e que não tiveram relação com pessoas do mesmo sexo por 66 (46,1\%) dos participantes (Tabela 3). 


\begin{tabular}{|c|c|c|}
\hline Variáveis & $\mathbf{N}$ & $\%$ \\
\hline \multicolumn{3}{|l|}{ Idade da primeira relação sexual } \\
\hline Não iniciou a vida sexual & 60 & 41,9 \\
\hline Aos 15 anos & 29 & 20,3 \\
\hline Aos 14 anos & 23 & 16,1 \\
\hline Aos 12 anos & 10 & 7,0 \\
\hline Aos 13 anos & 9 & 6,3 \\
\hline Aos 16 anos & 8 & 5,6 \\
\hline Aos 17 anos & 2 & 1,4 \\
\hline Não respondeu & 2 & 1,4 \\
\hline \multicolumn{3}{|c|}{ A pessoa com quem se teve a primeira relação } \\
\hline Ainda não iniciou a vida sexual & 60 & 39,2 \\
\hline Namorado(a) & 46 & 30,1 \\
\hline Não quero responder & 18 & 11,8 \\
\hline Pessoa com quem você ficou & 17 & 11,1 \\
\hline Marido ou companheiro & 12 & 7,8 \\
\hline \multicolumn{3}{|c|}{ Qual método contraceptivo é utilizado atualmente } \\
\hline Não iniciou a vida sexual & 60 & 41,9 \\
\hline Preservativo masculino & 38 & 26,6 \\
\hline Preservativo feminino & 37 & 25,9 \\
\hline Pílula anticoncepcional & 1 & 0,7 \\
\hline D.I.U. & 5 & 3,5 \\
\hline Coito interrompido & 2 & 1,4 \\
\hline \multicolumn{3}{|l|}{ Filhos } \\
\hline Não & 128 & 89,5 \\
\hline $\operatorname{Sim}$ & 15 & 10,5 \\
\hline \multicolumn{3}{|l|}{ Último parceiro (a) sexual } \\
\hline Vida sexual não iniciada & 60 & 41,9 \\
\hline Namorado(a) & 42 & 29,4 \\
\hline Parceiro(a) casual/ficante & 23 & 16,1 \\
\hline Outro & 13 & 9,1 \\
\hline Esposo(a) ou companheiro(a) regular & 5 & 3,5 \\
\hline \multicolumn{3}{|c|}{ Já teve relação sexual com pessoa do mesmo sexo } \\
\hline Sim & 14 & 9,8 \\
\hline Não & 66 & 46,1 \\
\hline Não teve relação com nenhum sexo & 60 & 42 \\
\hline Não quero responder & 3 & 2,1 \\
\hline
\end{tabular}

Fonte: Elaborado pelos autores, 2020.

Na tabela 4 encontram-se os resultados em relação à prática do uso do preservativo pelos adolescentes participantes. Constatou-se que 60 (41,9\%) adolescentes ainda não haviam tido a primeira relação sexual. Apenas $23(16,1 \%)$ referiram utilizar o preservativo em todas as relações sexuais. Dentre os motivos para o não uso do preservativo masculino, o estudo identificou que $27(18,9 \%)$ adolescentes colocaram confiança no parceiro como o principal motivo.

No que concerne à forma de utilizar o preservativo, a maior frequência foi de alunos que usavam o preservativo somente no final da relação sexual $39(27,3 \%)$ e apenas 29 (20,3\%) utilizavam o preservativo do início ao fim da relação sexual. O fato de "ter as preliminares" foi a resposta mais frequente para o não uso do preservativo desde o início da relação sexual, 37 (25,9\%). Sobre a utilização do preservativo nas últimas cinco relações sexuais, oito $(5,6 \%)$ afirmaram ter utilizado sempre, enquanto $35(24,5 \%)$ informaram nunca ter utilizado (Tabela 4$)$. 
Tabela 4. Práticas em relação ao uso do preservativo de adolescentes de escola estadual de ensino médio., Sobral-Ceará, 2019. (n=143)

\begin{tabular}{|c|c|c|}
\hline Variáveis & $\mathbf{N}$ & $\%$ \\
\hline \multicolumn{3}{|l|}{ Utiliza o preservativo durante as relações sexuais } \\
\hline As vezes & 27 & 18,9 \\
\hline Não iniciou a vida sexual & 60 & 41,9 \\
\hline Não quis responder & 5 & 3,5 \\
\hline Nunca & 28 & 19,6 \\
\hline Sempre & 23 & 16,1 \\
\hline \multicolumn{3}{|l|}{ Motivos da não utilização } \\
\hline Não iniciou a vida sexual & 60 & 41,9 \\
\hline Parceiro não gosta & 12 & 8,4 \\
\hline Confiança no parceiro & 27 & 18,9 \\
\hline Usa outro método contraceptivo & 12 & 8,4 \\
\hline O parceiro é conhecido ou fixo & 14 & 9,8 \\
\hline Outro & 18 & 12,6 \\
\hline \multicolumn{3}{|l|}{ Como você utiliza o preservativo? } \\
\hline Somente no final da relação sexual & 39 & 27,3 \\
\hline Não iniciou a vida sexual & 60 & 42 \\
\hline Do início ao fim da relação sexual & 29 & 20,3 \\
\hline Outro & 15 & 10,4 \\
\hline \multicolumn{3}{|c|}{ Motivos de não usar o preservativo desde o início da relação sexual } \\
\hline Não iniciou a vida sexual & 60 & 41,9 \\
\hline Porque tem as preliminares & 37 & 25,9 \\
\hline Não me sinto confortável & 26 & 18,2 \\
\hline Porque não é necessário & 16 & 11,2 \\
\hline Vergonha de comprar ou pedir no posto de saúde & 4 & 2,8 \\
\hline \multicolumn{3}{|c|}{$\begin{array}{l}\text { Nas últimas cinco relações sexuais, com qual frequência o preservativo foi } \\
\text { usado? }\end{array}$} \\
\hline Nunca & 35 & 24,5 \\
\hline Frequentemente & 16 & 11,2 \\
\hline Algumas vezes & 12 & 8,4 \\
\hline Raramente & 12 & 8,4 \\
\hline Sempre & 8 & 5,6 \\
\hline
\end{tabular}

Fonte: Elaborado pelos autores, 2020.

A Tabela 5 apresenta a classificação do conhecimento, da atitude e prática dos adolescentes participantes acerca do preservativo masculino, de acordo com os critérios do CAP preestabelecidos. Diante dos achados, evidenciouse que quase a totalidade dos adolescentes do estudo, 133 (9,0\%), apresentou conhecimento inadequado quanto ao preservativo masculino, bem como no tocante à atitude e prática, $105(73,4 \%)$ e $130(90,9 \%)$ adolescentes, respectivamente.

Tabela 5. Classificação do conhecimento, da atitude e prática de adolescentes de escola estadual de ensino médio acerca do preservativo masculino. Sobral-Ceará, 2019. $(n=143)$

\begin{tabular}{lcc}
\hline Variáveis & N & $\%$ \\
\hline Conhecimento & & 93,0 \\
Inadequado & 133 & 7,0 \\
Adequado & 10 & 73,4 \\
Atitude & 105 & 26,6 \\
Inadequado & 38 & 90,9 \\
Adequado & & 130 \\
Prática & 13 & 9,1 \\
Inadequado & & 9 \\
Adequado & & 9 \\
\hline
\end{tabular}

Fonte: Elaborado pelos autores, 2020. 


\section{Discussão}

Mediante os resultados expostos, corroborando com esta pesquisa, outro estudo realizado com adolescentes escolares em Fortaleza, Ceará, no ano de 2014, evidenciou que a média de idade entre eles é de 16 anos, tanto nas escolas privadas como públicas, e que a maioria era solteiros e heterossexuais, além de também residirem com os pais ${ }^{17}$. Desta forma, os resultados contribuem para melhor compreensão do perfil dos estudantes do estudo e o planejamento de ações de educação em saúde.

Percebe-se que o diálogo com os pais sobre a temática da sexualidade ainda é um tabu, representando assim uma preocupação, pois, em pleno século XXI, a sociedade ainda carrega a marca do pudor extremo das gerações passadas, em que falar sobre sexo pode despertar estruturas de culpa, vergonha, sendo melhor calar do que ensinar, o que é discutido em outro estudo que objetivou mudar as normas e atitudes em relação à comunicação acerca da sexualidade dentro das famílias ${ }^{18}$.

Nesse contexto, existem muitos fatores que podem agravar a fase da adolescência, devido ser uma fase do início da atividade sexual, os adolescentes se tornam mais vulneráveis a riscos, como IST/Aids ou gravidez indesejada, visto que muitos não têm os conhecimentos necessários em relação aos riscos que estão sujeitos e, consequentemente, não se previnem de forma adequada ${ }^{19}$.

De modo geral, por se sentirem jovens e saudáveis, os adolescentes pouco frequentam os serviços de saúde, o que vai de encontro com estudo realizado com adolescentes de 11 a 16 anos, que quando questionados sobre o conceito de Estratégia Saúde da Família, julgaram que era designado apenas para o tratamento de doenças, o que gera limitação na busca dos serviços por esse público, uma vez que também consideram que este campo de atenção à saúde é relevante apenas para esses fins ${ }^{20}$.

Desse modo, notou-se também que há carência em relação a informações sobre saúde sexual reprodutiva por professores. Assim, é necessário que se aprofunde mais, considerando que nesta fase a maioria está frequentando a escola, portanto, este é um importante local onde pode haver orientação. Nesse contexto, escola e professores se configuram como grandes aliados, para que se possam romper as barreiras do silêncio, do medo e da culpa que giram em torno do tema sexualidade ${ }^{19}$. Assim, atividade educativa na escola deve ser implementada e fomentada, no intuito de contribuir para prática sexual futuramente segura.

Os achados se mostram preocupantes, visto que há vários motivos indicados como respostas para não utilização dos preservativos, sendo a conscientização frente a essas causas ainda um grande desafio. Desta forma, é fundamental a parceria entre as áreas de educação e saúde para substituir essas ideias por conhecimento adequado, de forma a contribuir com uma atividade sexual segura. Essa articulação tem grande utilidade para prevenir, de forma mais específica, problemas evidenciados nessa fase da vida que é tão propícia a riscos $\stackrel{19}{ }$.

Merece destaque o fato de $27,3 \%$ dos adolescentes deste estudo não acharem necessário o uso do preservativo para a realização de sexo anal. A literatura aponta que uma das formas de contágios e riscos de contrair HIV/Aids é por meio do sexo anal, principalmente pelas baixas taxas de uso do preservativo durante esta relação, e ainda mais pela prática inconsciente de pessoas soropositivas, fazendo com que um soro negativo seja receptivo de forma desprotegida ${ }^{21}$.

Estudo realizado em uma Rede Pública Estadual de Ensino da Região Oeste de Goiânia, no ano de 2015, com 210 adolescentes apontou que eles ficam mais propensos a vulnerabilidades, pois o início da atividade sexual é imaturo, proporcionando a participação nas relações de diferentes parceiros ${ }^{22}$.

Ensaio feito na Europa e na América do Norte, Global School-Based Student Health Survey - GSHS, pela OMS, no ano de 2010, demonstrou que a prática da sexualidade é desempenhada principalmente aos 15 anos, e destacou que $26 \%$ dos escolares já haviam praticado sexo nesse faixa etária ${ }^{23}$, o que pode ser comparado ao presente estudo, que apresentou resultado quase semelhante, visto que $29 \%$ dos adolescentes tiveram a primeira prática sexual nessa referida idade. 
Alerta-se para o fato de que a gravidez na adolescência é indício de preocupação, pois é nessa fase da vida que o indivíduo está passando por diversas mudanças corporais, sendo o período em que o corpo está se preparando para diversas transformações em decorrência do próprio desenvolvimento. Além disso, nessas situações, tem-se o afastamento da adolescente do convívio escolar, devido ao surgimento de outras responsabilidades. Ademais, em muitos casos, os projetos pessoais são interrompidos, gerando, assim, consequências sociais, acrescenta-se risco à saúde, visto que antes dos 18 anos de idade a gestação é de alto risco, em decorrência do próprio desenvolvimento $\underline{24}$.

Destarte, as instruções voltadas para o período de inicialização da prática sexual devem ser enfocadas nas políticas de saúde pública, pois conforme a Organização Pan-Americana da Saúde, as políticas voltadas para a adolescência ainda não são suficientes, apesar de haver histórico importante, no que se diz respeito a ações voltadas para o atendimento de saúde sexual dos adolescentes. Nesse contexto, o objetivo é que se possa criar alternativas para ampliação dessa atenção, de forma integral e universal, por meio de acordos internacionais ou intermédio, pelo Ministério da Saúde brasileiro ${ }^{20}$.

Revisão narrativa sobre o uso do preservativo masculino no Brasil, em diferentes grupos populacionais, e fatores associados ao uso observou que apesar de haver muitas informações disponíveis sobre a temática da sexualidade na adolescência, ainda existe lacuna entre conhecimento e prática sobre o uso do preservativo ${ }^{25}$, o que corrobora com o atual estudo que observou altas taxas de adolescentes com conhecimento inadequado, atitude e prática quanto ao uso do preservativo masculino.

Os resultados do estudo se mostram preocupantes, haja vista que os adolescentes nessa fase da vida iniciam cada vez mais cedo a prática sexual, ainda de maneira insegura, pelo não uso ou uso inadequado do preservativo masculino, estando sujeitos às IST/ HIV/Aids e gravidez indesejada. Diante disso, o conhecimento adequado sobre o uso do preservativo masculino é imprescindível para uma prática sexual adequada. Todavia, o conhecimento, isoladamente, não é um fator determinante, considerando que o comportamento envolve muitos outros fatores, como os sociais e os culturais.

Dentre as limitações do estudo, destacam-se os questionários aplicados em um único encontro, pouco tempo disponível para o preenchimento, haja vista que, no período da coleta dos dados, os alunos estavam em intensa jornada de estudos para provas escolares. Dessa forma, pode ter levado os participantes a ler o questionário rapidamente e com menos foco nas questões, o que pode ter implicado nos resultados obtidos. Salienta-se que havendo maior disponibilidade de tempo para aplicação em cada sala, haveria mais tranquilidade na marcação dos itens, além disso, o aumento da quantidade de encontros facilitaria a divisão das partes do questionário, tornando a leitura menos massiva para resolução das questões.

Cita-se também possível viés de informação, por serem questões que abordem a sexualidade e, consequentemente, envolvem tabus, mitos, medos e vergonha entre os adolescentes.

\section{Conclusão}

O estudo oportunizou identificar o conhecimento, a atitude e a prática de adolescentes escolares em relação ao uso do preservativo masculino. Percebeu-se, por meio dos resultados obtidos, fragilidade no diálogo entre adolescentes e pais sobre saúde sexual e reprodutiva. Na escola, esse diálogo se apresentou mais frequente, porém, ainda, é preciso ampliar essa discussão.

As fragilidades nas informações obtidas frente à temática estudada refletem o conhecimento inadequado pela quase totalidade dos adolescentes, seguidas pela atitude e prática inadequada quanto ao uso do preservativo masculino. Os adolescentes desconheciam os cuidados para utilização dos preservativos, não utilizavam da maneira e frequência adequada, evidenciando, assim, fator preocupante para saúde pública. 
Diante do exposto, é crucial que sejam reforçadas as práticas educativas em saúde sexual e reprodutiva para os adolescentes, privilegiando locais em que os adolescentes estejam inseridos, principalmente as escolas. Essa prática deve abranger equipes multiprofissionais, como professores, coordenadores, além de vinculação com profissionais da saúde, especialmente da atenção primária em saúde, visto que é a que está mais próxima da comunidade.

Destaca-se o papel de enfermeiros na promoção de orientações aos pais sobre saúde sexual e reprodutiva, para que estes possam ir aos poucos rompendo as barreiras do medo, do silêncio e da vergonha de tratar desta temática com os filhos.

Assim, salienta-se a importância de mais estudos sobre a temática saúde sexual e reprodutiva no âmbito escolar, pois o ambiente é oportuno para o diálogo com o adolescente, bem como para realização de ações em saúde. Diante disto, urge potencializar estratégias educativas, com intuito de ampliar o conhecimento, a atitude e a prática desse público em relação ao uso do preservativo masculino.

\section{Contribuições dos autores}

Aragão JMN participou da concepção do projeto, delineamento, busca e análise dos dados da pesquisa, interpretação dos resultados, redação e revisão final do artigo científico. Amaral HRM participou do delineamento, coleta e análise de dados da pesquisa, interpretação dos resultados e redação do artigo científico. Sousa FWM participou da busca e análise dos dados da pesquisa, interpretação dos resultados e redação do artigo científico. Vasconcelos MIO e Oliveira EN participaram da interpretação dos resultados, redação e revisão do artigo científico.

\section{Conflitos de interesses}

Nenhum conflito financeiro, legal ou político envolvendo terceiros (governo, empresas e fundações privadas, etc.) foi declarado para nenhum aspecto do trabalho submetido (incluindo, mas não se limitando a subvenções e financiamentos, participação em conselho consultivo, desenho de estudo, preparação de manuscrito, análise estatística, etc.).

\section{Referências}

1. Cavalcante FML, Sousa FWM, Oliveira IKM, Amaral HRM, Rosa BSC, Gomes JS, et al. Atividades de extensão universitária: um olhar para promoção da saúde do adolescente. Saúde Redes [Internet]. 2019;5(3):305-15. Disponível em: https://pesquisa. bvsalud.org/portal/resource/pt/biblio-1116471

2. Lei 8.069 , de 13 de julho de 1990 (Brasil). Dispõe sobre o Estatuto da Criança e do Adolescente e dá outras providências. Estatuto da Criança e do adolescente. Brasília: Ministério da justiça. 1990. Disponível em: https://legislacao.presidencia.gov.br/atos/?tipo $=$ LEl\&numero $=8069 \&$ ano $=1990 \&$ ato $=461 \mathrm{cXRq1}$ keFpWT13a

3. Kerntopf MR, Lacerda JFE, Fonseca NH, Nascimento EP, Lemos IZC, Fernandes GP. Sexualidade na adolescência: uma revisão crítica da literatura. Rev Adolesc. Saúde [Internet]. 2016;13(supl.2)106-13. Disponível em: http://www. adolescenciaesaude.com/detalhe artigo.asp?id=590\#

4. Aragão JMN, Gubert FA, Torres RAM, Silva ASR, Vieira NFC. O uso do Facebook na aprendizagem em saúde: percepções de adolescentes escolares. Rev Bras Enferm. 2018;71(2):265-71. https://doi.org/10.1590/0034-7167-2016-0604

5. Moraes GM, Schimin ES. Os desafios da escola pública paranaense na perspectiva do professor de PDE [Internet]. Vol 1. Paraná: Governo do Estado; 2016. Disponível em: http://www.diaadiaeducacao.pr.gov.br/portals/cadernospde/ pdebusca/producoes_pde/2016/2016_artigo_cien_unicentro. gildomarcosmoraes.pdf

6. Araújo RLD, Rodrigues ESRC, Oliveira GG, Sousa MO. Gravidez na adolescência: consequências centralizadas para a mulher. Temas em saúde [Internet]. 2016;16(2):567-87. Disponível em: https://temasemsaude.com/wp-content/uploads/2016/08/16231. pdf

7. Silva JMB, Ferreira JA, Enders BC, Meneses RMV. Percepção de adolescentes grávidas acerca de sua gestação. Revista Baiana de Enfermagem [Internet]. 2011;25(1):23-32. Disponível em: https://portalseer.ufba.br/index.php/enfermagem/article/ download/5234/4324

8. Azevedo AEBI, Eisenstein E, Bermudez BEBV, Fernandes EC, Oliveira HF, Hagel LD, et al. Guia Prático de Atualização: prevenção da gravidez na adolescência. Adolesc. Saude [Internet]. 2018;15(1):86-94. Disponível em: https://cdn.publisher.gn1.link/ adolescenciaesaude.com/pdf/v15s1a11.pdf

9. Almeida RAAS, Corrêa RGCF, Rolim TP, Hora JM, Linard AG, Coutinho NPS, et al. Conhecimento de adolescentes relacionados às doenças sexualmente transmissíveis e gravidez. Rev Bras Enferm. 2017;70(5):1087-94. http://dx.doi.org/10.1590/0034-71672016-0531 
10. Molina MCC, Stoppiglia PGS, CBG Martins, Alencastro LCS. Conhecimento de adolescentes do ensino médio quanto aos métodos contraceptivos. O Mundo da Saúde [Internet]. 2015;39(1):22-31. Disponível em: http://bvsms.saude.gov.br/bvs/ periodicos/mundo_saude_artigos/Conhecimento_adolescentes ensino.pdf

11. Panneflex PL, Salazar DPA, Munive MV. Conocimientos, creencias y prácticas de los adolescentes de la cultura Caribe en anticoncepción. Rev Cuid. 2016;7(1):1204-9. http://dx.doi. org/10.15649/cuidarte.v7i1.243

12. Vieira1 EL, Pessoa GRS, Vieira LL, Carvalho WRC, Firmo WCA. Uso e conhecimento sobre métodos contraceptivos de estudantes da rede de ensino pública e privada do município de Bacabal-MA. Revista Científica do ITPAC [Internet]. 2016;9(2):88-107. Disponível em: https://assets.unitpac.com.br/arquivos/Revista/78/Artigo_10. pdf

13. Ministério da Saúde (Brasil), Secretaria de Atenção em Saúde, Departamento de Ações Programáticas Estratégicas. Diretrizes nacionais para a atenção integral à saúde de adolescentes e jovens na promoção, proteção e recuperação da saúde. / Ministério da Saúde [Internet]. Brasília: Ministério da Saúde; 2010. p.132. Disponível em: https://bvsms.saude.gov.br/bvs/ publicacoes/diretrizes_nacionais_atencao_saude_adolescentes jovens_promocao_saude.pdf

14. World Health Organization. Nutrition in adolescence - issues and challenges for the health sector: issues in adolescent health and development [Internet]. Geneva: World Health Organization; 2005. Disponível em: https://apps.who.int/iris/ handle/10665/43342

15. Nicolau Al. Conhecimento, Atitude e Prática de presidiárias quanto ao uso do preservativo masculino e feminino [dissertação] [Internet]. Fortaleza: Universidade Federal do Ceará; 2010. Disponível em: http://www.repositorio.ufc.br/handle/riufc/1917

16. Ministério da Educação (Brasil). Instituto Nacional de Desenvolvimento da Educação. Manual aplicador do estudo CAP. Brasília: Ministério da Saúde; 2002.

17. Gubert FA, Vieira NFC, Costa RLC, Malta EFGD, Fonseca IS, Brito LLMS, et al. Perfil sociodemográfico e sexual de adolescentes escolares sexualmente ativas em Fortaleza-CE. Adolesc Saude [Internet]. 2016;13(supl.2):41-50. Disponível em: https://cdn. publisher.gn1.link/adolescenciaesaude.com/pdf/v13s2a06.pdf
18. Frederico M, Arnaldo C, Michielsen K, Decat P. Adult and young women communication on sexuality: a pilot intervention in Maputo-Mozambique. Reprod Health. 2019;16(144):1-12. https:// doi.org/10.1186/s12978-019-0809-3

19. Sasaki RSA, Leles CR, Malta DC, Sardinha LMV, Freire MCM. Prevalência de relação sexual e fatores associados em adolescentes escolares de Goiânia, Goiás, Brasil. Ciênc. saúde coletiva. 2015;20(1):95-104. https://doi.org/10.1590/1413$\underline{81232014201.06332014}$

20. Alves MJ, Albuquerque GA, Silva AS, Belém JM, Nunes JF, Leite MF, et al. Fatores envolvidos na adesão de estudantes adolescentes à estratégia saúde da família. SANARE [Internet]. 2016;15(2):37-46. Disponível em: https://sanare.emnuvens.com. br/sanare/article/view/1036/582

21. Dourado I, MacCarthy S, Reddy M, Calazan G, Gruskin S. Revisitando o uso do preservativo no Brasil. Rev. bras. epidemiol., 2015;18(supl.1):63-88.https://doi.org/10.1590/18094503201500050006

22. Soares LR, Cabero FV, Souto TG, Coelho RFS, Lacerda LCM, Matão LMELM. Avaliação do comportamento sexual entre jovens e adolescentes de escolas públicas. Adolesc. Saude [Internet]. 2015;12(2):76-84. Disponível em: http://adolescenciaesaude.com/ detalhe_artigo.asp?id=504

23. Currie C, Zanotti C, Morgan A, Curie D, Looze M, Roberts C, et al. (Eds.). Social determinants of health and well-being among young people: Health Behaviour in School-Aged Children (HBSC) study: international report from the 2009/2010 survey [Internet]. Copenhagen: World Health Organization; 2012. Disponível em: https://www.euro.who.int/ data/assets/pdf file/0003/163857/ Social-determinants-of-health-and-well-being-among-youngpeople.pdf

24. Santos RCAN, Silva RM, Queiroz MVO, Jorge HMF, Brilhante AVM. Realidades e perspectivas de mães adolescentes acerca da primeira gravidez. Rev. Bras. Enferm. 2017;71(1):65-72. https://doi. org/10.1590/0034-7167-2016-0444

25. Hiltabiddle SJ. Adolescent condom use, the health belief model, and the prevention of sexually transmitted disease. J Obstet Gynecol Neonatal Nurs. 1996;25(1):61-6. https://doi. org/10.1111/j.1552-6909.1996.tb02514.X 